\title{
A LOOK AT THE FAITH CONJECTURE
}

\author{
PERE ARA \\ Departament de Matemàtiques, Universitat Autònoma de Barcelona, (08193) Bellaterra (Barcelona), Spain \\ e-mail:para@mat.uab.es
}

\author{
W. K. NICHOLSON \\ Department of Mathematics, University of Calgary, Calgary T2N 1N4, Canada \\ e-mail:wknichol@ucalgary.ca \\ and M. F. YOUSIF \\ Department of Mathematics, Ohio State University, Lima, Ohio 45804, USA \\ e-mail:yousif.1@osu.edu
}

(Received 10 December, 1998)

\begin{abstract}
A well known result of B. Osofsky asserts that if $R$ is a left (or right) perfect, left and right selfinjective ring then $R$ is quasi-Frobenius. It was subsequently conjectured by Carl Faith that every left (or right) perfect, left selfinjective ring is quasi-Frobenius. While several authors have proved the conjecture in the affirmative under some restricted chain conditions, the conjecture remains open even if $R$ is a semiprimary, local, left selfinjective ring with $J(R)^{3}=0$. In this paper we construct a local ring $R$ with $J(R)^{3}=0$ and characterize when $R$ is artinian or selfinjective in terms of conditions on a bilinear mapping from a $D$ - $D$-bimodule to $D$, where $D$ is isomorphic to $R / J(R)$. Our work shows that finding a counterexample to the Faith conjecture depends on the existence of a $D$ - $D$-bimodule over a division ring $D$ satisfying certain topological conditions.
\end{abstract}

1991 Mathematics Subject Classification. 16D21, 16D50, 16L30, 16L60, 16P20.

A ring $R$ is called quasi-Frobenius if it is left and right artinian and left and right selfinjective, equivalently, if $R$ has the ACC on right or left annihilators and is right or left selfinjective. The Faith conjecture (see [4] or [5]) asserts that every left or right perfect, right selfinjective ring $R$ is quasi-Frobenius. Following ideas of Osofsky [10], we construct a local ring $R$ with $J(R)^{3}=0$ and characterize when $R$ is artinian or selfinjective in terms of conditions on a bilinear mapping from a $D$ - $D$-bimodule to a division ring $D \cong R / J(R)$. We conclude by characterizing other properties of $R$ in a similar way.

Throughout this paper all rings are associative with unity, and all modules are unital. If $R$ is a ring we write $J=J(R)$ for the Jacobson radical of $R$. The socle of a module $M$ is denoted by $\operatorname{soc}(M)$. Annihilators of a subset $X \subseteq R$ are written $l(X)=\{a \in R \mid a X=0\}$ and $r(X)=\{a \in R \mid X a=0\}$. We write $N \subseteq \subseteq^{e s s} M$ (respectively $N \subseteq \max M$ ) to indicate that $N$ is an essential (maximal) submodule of $M$. The symbol $D$ will always denote a division ring.

Generalities. If $S$ is any ring and ${ }_{S} V_{S},{ }_{S} W_{S}$ and ${ }_{S} P_{S}$ are bimodules, a function $V \times W \rightarrow P$, which we write multiplicatively as $(v, w) \rightarrow v w$, is called a bimap if

(1) $\left(v+v_{1}\right) w=v w+v_{1} w$ and $(s v) w=s(v w)$,

(2) $v\left(w+w_{1}\right)=v w+v w_{1}$ and $v(w s)=(v w) s$,

(3) $(v s) w=v(s w)$ 
hold for all $v, v_{1}$ in $V$, all $w, w_{1}$ in $W$, and all $s$ in $S$. This is equivalent to the existence of a $S$-S-bimodule map $V \otimes_{S} W \rightarrow P$. Our interest is in the case when $S=D$ is a division ring.

Definition. Let ${ }_{D} V_{D}$ and ${ }_{D} P_{D}$ be nonzero bimodules over a division $\operatorname{ring} D$, and suppose that a bimap $V \times V \rightarrow P$ is given. Write

$$
R=[D, V, P]=D \oplus V \oplus P
$$

and define a multiplication on $R$ by

$$
(d+v+p)\left(d_{1}+v_{1}+p_{1}\right)=d d_{1}+\left(d v_{1}+v d_{1}\right)+\left(d p_{1}+v v_{1}+p d_{1}\right) .
$$

It is a routine verification that $R$ is an associative ring if and only if the product $V \times V \rightarrow P$ is a bimap. The ring $R$ has a matrix representation as

$$
R=\left\{\left[\begin{array}{ccc}
d & v & p \\
0 & d & v \\
0 & 0 & d
\end{array}\right] \mid d \in D, v \in V \text { and } p \in P\right\}
$$

Note that we shall assume that $V \neq 0$ and $P \neq 0$ throughout this paper.

Our first result collects several properties of this ring that will be used frequently below. If $X$ is a nonempty subset of $V$ we write $l_{V}(X)=\{v \in V \mid v X=0\}$ and $r_{V}(X)=\{v \in V \mid X v=0\}$.

Lemma 1 . The ring $R=[D, V, P]$ has the following properties.

(1) $R$ is an associative ring.

(2) $V P=P V=P^{2}=0$.

(3) $R$ is local, $J=V \oplus P, J^{2}=V^{2} \subseteq P$ and $J^{3}=0$.

(4) $\operatorname{soc}\left(R_{R}\right)=l(J)=l_{V}(V) \oplus P \subseteq \subseteq^{e s s} R_{R}$.

(5) $x R=x D$, for all $x \in \operatorname{soc}\left(R_{R}\right)$.

(6) If $X_{D} \subseteq V$, then $X \oplus P$ is a right ideal of $R$; and every right ideal $T$ such that $P \subseteq T \subseteq J$ has this form.

(7) Every right $D$-subspace of $\operatorname{soc}\left(R_{R}\right)$ is a right ideal of $R$.

(8) Let $X$ and $Y$ be right $D$-subspaces of $\operatorname{soc}\left(R_{R}\right)$. Then every D-linear transformation $X \rightarrow Y$ is R-linear.

Proof. (1) and (2) are routine verifications.

(3). The map $(d+v+p) \mapsto d$ is a ring morphism from $R$ onto $D$ with kernel $V \oplus P$, proving that $R$ is local and $J=V \oplus P$. The rest of (3) is easily checked.

(4). We have $\operatorname{soc}\left(R_{R}\right) \subseteq{ }^{e s s} R_{R}$ because $R$ is semiprimary by (3), and $\operatorname{soc}\left(R_{R}\right)=l(J)$ because $R$ is semilocal. Now

$$
l(J)=\{d+v+p \mid d V=0 \text { and } d P+v V=0\} .
$$

Since $V \neq 0$ it follows that $d=0$, whence $v V=0$. Thus $l(J) \subseteq l_{V}(V) \oplus P$. The other inclusion is clear.

(5). If $x=v+p \in \operatorname{soc}\left(R_{R}\right)$, where $v V=0$, then $x R=\{v d+p d \mid d \in D\}=x D$.

(6). It is routine that $X \oplus P$ is a right ideal. Given $P \subseteq T \subseteq J$, we have $T=(T \cap V) \oplus P$ by the modular law. 
(7). This is a direct calculation using $\operatorname{soc}\left(R_{R}\right)=l_{V}(V) \oplus P$ from (4).

(8). If $r=d+v+p$ then $x r=x d$, for all $x \in X \cup Y$, by (2) and (4).

Note that Lemma 1(5) shows that a right ideal $T \subseteq \operatorname{soc}\left(R_{R}\right)$ is simple if and only if $\operatorname{dim}_{D}\left(T_{D}\right)=1$. The next result shows that if $\operatorname{dim}\left(P_{D}\right)=1$ we can obtain the converse to (6) and (7) of Lemma 1, and so characterize the right ideals of $R=[D, V, P]$. Call a right ideal $T \subseteq R$ proper if $T \neq R$.

Lemma 2. Let $R=[D, V, P]$, where $\operatorname{dim}\left(P_{D}\right)=1$. Then the proper right ideals of $R$ are

$$
\left\{X \oplus P \mid X_{D} \subseteq V\right\} \text { and }\left\{Y \mid Y_{D} \subseteq \operatorname{soc}\left(R_{R}\right)\right\}
$$

Proof. These are all right ideals by (6) and (7) of Lemma 1 . If $T \neq R$ is a right ideal, then $T \subseteq J$ because $R$ is local. Since $P_{R}$ is simple, either $P \subseteq T$ or $P \cap T=0$. In the first case, $T=X \oplus P$ for $X_{D} \subseteq V$ by Lemma 1(6). If $P \cap T=0$, we show that $T \subseteq \operatorname{soc}\left(R_{R}\right)$. If $t=v+p \in T$ then, for $v_{1} \in V, v v_{1}=(v+p) v_{1} \in P \cap T=0$. Thus $v \in l_{V}(V)$, and so $t \in l_{V}(V) \oplus P=\operatorname{soc}\left(R_{R}\right)$.

Note that, under the hypotheses of Lemma 2, the proper (two-sided) ideals of $R$ are $\left\{X \oplus P \mid{ }_{D} X_{D} \subseteq V\right\}$ and $\left\{Y \mid{ }_{D} Y_{D} \subseteq\left[l_{V}(V) \cap r_{V}(V)\right] \oplus P\right\}$.

Even without the hypothesis that $\operatorname{dim}\left(P_{D}\right)=1$ we can characterize when $R=[D, V, P]$ is right artinian.

Proposition 1. The following conditions are equivalent for $R=[D, V, P]$.

(1) $R$ is right artinian.

(2) $R$ is right noetherian.

(3) $\operatorname{dim}\left(V_{D}\right)<\infty$ and $\operatorname{dim}\left(P_{D}\right)<\infty$.

(4) $\operatorname{dim}\left(R_{D}\right)<\infty$.

Proof. The implications $(3) \Rightarrow(4) \Rightarrow(1) \Rightarrow(2)$ are clear. If $R_{R}$ is noetherian and $X_{1} \subset X_{2} \subset \cdots$ are subspaces of $V_{D}$, then $X_{1} \oplus P \subset X_{2} \oplus P \subset \cdots$. It follows from Lemma 1(6) that $\operatorname{dim}\left(V_{D}\right)<\infty$. We have $\operatorname{dim}\left(P_{D}\right)<\infty$ because every $D$-subspace of $P$ is a right ideal (by Lemma 1(7)).

The main theorem. In order to study the Faith conjecture, we must characterize when $R=[D, V, P]$ is right selfinjective. We begin by characterizing a weaker injectivity condition. A ring $R$ is called right mininjective if every $R$-morphism $\gamma$ from a simple right ideal to $R_{R}$ is given by left multiplication $\gamma=c$. by an element $c$ of $R$, equivalently [8, Lemma 1.1] if $\operatorname{lr}(k)=R k$ whenever $k R$ is a simple right ideal of $R$. Clearly every right selfinjective ring is right mininjective. The next result will be used several times.

Proposition 2. The following are equivalent for $R=[D, V, P]$.

(1) $R$ is right mininjective.

(2) $l_{V}(V)=0$ and $\operatorname{dim}\left({ }_{D} P\right)=1$.

Proof. (1) $\Rightarrow(2)$. If $0 \neq p_{\circ} \in P$ and $u \in l_{V}(V)$, and if $\gamma: p_{\circ} D \rightarrow\left(u+p_{\circ}\right) D$ is given by $\gamma\left(p_{\circ} d\right)=\left(u+p_{\circ}\right) d$, then $\gamma$ is $R$-linear by Lemma $1(8)$. By $(1), \gamma=c$. is left multi- 
plication by $c \in R$ and so $u+p_{\circ}=\gamma\left(p_{\circ}\right)=c p_{\circ} \in P$. Thus $u=0$, whence $l_{V}(V)=0$. If $0 \neq p \in P$, then $p R=p D$ is simple so that $\operatorname{lr}(p)=R p$ by (1). Hence Lemma 1(4) gives

$$
D p=R p=\operatorname{lr}(p)=l(J)=l_{V}(V) \oplus P=P .
$$

Thus $\operatorname{dim}\left({ }_{D} P\right)=1$.

(2) $\Rightarrow(1)$. Let $\gamma: K_{R} \rightarrow R_{R}$ be $R$-linear, where $K_{R}$ is a simple right ideal; we must show that $\gamma=c$. for $c \in R$. We may assume that $\gamma \neq 0$. We have $\operatorname{soc}\left(R_{R}\right)=P$ by (2), and so $K \subseteq P$. It follows from Lemma $1(7)$ that $\operatorname{dim}\left(K_{D}\right)=1$. Write $K=p_{\circ} D$, where $p_{\circ} \in P$. Since $\gamma(K)$ is simple we have $\gamma(K) \subseteq \operatorname{soc}\left(R_{R}\right)=P=D p_{\circ}$ by (2); say $\gamma\left(p_{\circ}\right)=d_{\circ} p_{\circ}$, where $d_{\circ} \in D$. Then, for all $d \in D$,

$$
\gamma\left(p_{\circ} d\right)=\gamma\left(p_{\circ}\right) d=\left(d_{\circ} p_{\circ}\right) d=d_{\circ}\left(p_{\circ} d\right) .
$$

This shows that $\gamma=d_{\circ} \cdot$, as required. 1 give

It is worth noting that, since we are assuming that $P \neq 0,(4)$ and (7) of Lemma $\operatorname{soc}\left(R_{R}\right)$ is simple as a right ideal if and only if $l_{V}(V)=0$ and $\operatorname{dim}\left(P_{D}\right)=1$.

The condition that $\operatorname{dim}\left(P_{D}\right)=1$ holds if $R=[D, V, P]$ satisfies another important weakened form of selfinjectivity. A ring $R$ is called right simple-injective if every $R$ linear map with simple image from a right ideal of $R$ to $R$ is given by left multiplication by an element of $R$. Clearly every right simple-injective ring is right mininjective. The next lemma will be used later and strengthens the condition in Proposition 2.

Lemma 3. Suppose that the ring $R=[D, V, P]$ is right simple-injective. Then

$$
l_{V}(V)=0 \text { and } \operatorname{dim}\left(P_{D}\right)=1=\operatorname{dim}\left({ }_{D} P\right) .
$$

Proof. Since $R$ is right mininjective, $l_{V}(V)=0$ and $\operatorname{dim}\left({ }_{D} P\right)=1$, by Proposition 2. Suppose that $\operatorname{dim}\left(P_{D}\right) \geq 2$ and let $\left\{p_{1}, p_{2}, \cdots\right\}$ be a $D$-basis of $P_{D}$. Define $\alpha: P_{D} \rightarrow P_{D}$ by $\alpha\left(p_{1}\right)=p_{2}$ and $\alpha\left(p_{i}\right)=0$ for all $i \geq 2$. Then $\alpha$ is $R$-linear by Lemma 1(8) and so, since $\operatorname{im}(\alpha)=p_{2} D$ is simple, $\alpha=a$. for some $a \in R$ by hypothesis. If $a=d+v+p$, then $\alpha\left(p_{i}\right)=a p_{i}=d p_{i}$, for each $i$, so that $d=0$ because $\alpha\left(p_{2}\right)=0$. But then $p_{2}=\alpha\left(p_{1}\right)=d p_{1}=0$, a contradiction.

The condition in Lemma 3 does not characterize when $R=[D, V, P]$ is right simple-injective. This is part of our main result, a characterization of when $R=[D, V, P]$ is right selfinjective. Surprisingly, this is equivalent to simple-injectivity. The following "separation" axiom will be referred to several times.

Condition $S$. If $V=x D \oplus M_{D}, x \neq 0$, there exists $v_{\circ} \in V$ such that $v_{\circ} x \neq 0$ and $v_{\circ} M=0$.

Observe that Condition $\mathrm{S}$ is equivalent to asking that, if $x \in V-X$, where $X_{D} \subseteq V$ is any subspace, there exists $v_{\circ} \in V$ such that $v_{\circ} x \neq 0$ and $v_{\circ} X=0$. 
Theorem 1. Let $R=[D, V, P]$. The following are equivalent.

(1) $R$ is right selfinjective.

(2) $R$ is right simple-injective.

(3) $l_{V}(V)=0, \operatorname{dim}\left(P_{D}\right)=1=\operatorname{dim}\left({ }_{D} P\right)$, and Condition $S$ holds.

Proof. $(1) \Rightarrow(2)$. This is clear.

$(2) \Rightarrow(3)$. By Lemma 3 it remains to prove Condition S. Fix $0 \neq q \in P$ and let $V_{D}=x D \oplus M$, where $x \neq 0$ and $M \subseteq V_{D}$. Define

$$
\beta: V \oplus P=x D \oplus M \oplus P \rightarrow P \text { by } \beta(x d+m+p)=q d .
$$

This is well defined because $D$ is a division ring, and it is $R$-linear because

$$
\begin{aligned}
\beta\left[(x d+m+p)\left(d_{1}+v_{1}+p_{1}\right)\right] & =\beta\left[x d d_{1}+m d_{1}+\left(x d p_{1}+m v_{1}+p d_{1}\right)\right] \\
& =q\left(d d_{1}\right) \\
& =q d\left(d_{1}+v_{1}+p_{1}\right) \\
& =[\beta(x d+m+p)]\left(d_{1}+v_{1}+p_{1}\right) .
\end{aligned}
$$

Since $\beta[V \oplus P]=q D$ is simple, it follows from (2) that $\beta=b$. is left multiplication by $b \in R$. Write $b=d_{\circ}+v_{\circ}+p_{\circ}$, so that $q=\beta(x)=b x=d_{\circ} x+v_{\circ} x$. Hence $v_{\circ} x=q \neq 0$ and $d_{\circ} x=0$. This means that $d_{\circ}=0$, and so $v_{\circ} m=b m=\beta(m)=0$, for all $m \in M$, proving Condition $\mathrm{S}$.

(3) $\Rightarrow(1)$. If $T \subseteq R$ is a right ideal, let $\alpha: T \rightarrow R_{R}$ be $R$-linear; we must show that $\alpha=a$. for some $a \in R$. This is clear if $T=R$ or $T=0$. Assume $0 \subset T \subseteq J$. Since $\operatorname{soc}\left(R_{R}\right)=l_{V}(V) \oplus P=P$ is simple, by (3), it follows from Lemma 2 that

$$
T=X \oplus P
$$

for some $X_{D} \subseteq V$ because $T \neq 0$. Since $R$ is right mininjective by Proposition 2, $\alpha_{\mid P}=a \cdot$ for some $a \in R$.

Claim. If $x \in X$ then $\alpha(x)-a x \in P$.

Proof. Write $\alpha(x)=d_{1}+v_{1}+p_{1}$. If $v \in V$ is arbitrary, we have $x v \in P$ and so

$$
a(x v)=\alpha(x v)=\alpha(x) v=\left(d_{1}+v_{1}+p_{1}\right) v=d_{1} v+v_{1} v .
$$

As $a(x v)$ and $v_{1} v$ are in $P$, it follows that $d_{1} v=0$ and $a(x v)=v_{1} v$. Hence $d_{1}=0$ and $a x-v_{1} \in l_{V}(V)=0$. Thus $\alpha(x)=a x+p_{1}$, proving the Claim.

Now define $\beta: T \rightarrow R$ by $\beta=\alpha-a \cdot$. It suffices to show that $\beta=b \cdot$, for some $b \in R$ (because then $\alpha=(a+b) \cdot$ ). We have $P \subseteq \operatorname{ker}(\beta)$ because $\alpha_{\mid P}=a \cdot$, and so $\beta(T)=\beta(X \oplus P)=\beta(X) \subseteq P$ by the Claim. If $\beta=0$, take $b=0$. If $\beta \neq 0$ then $\beta(T)=P$ because $\operatorname{dim}\left(P_{D}\right)=1$, and the fact that $P \subseteq \operatorname{ker}(\beta) \subseteq X \oplus P$ gives $\operatorname{ker}(\beta)=Y \oplus P$ where $Y=X \cap \operatorname{ker}(\beta)$. Hence

$$
\frac{X}{Y} \cong \frac{X \oplus P}{Y \oplus P}=\frac{T}{\operatorname{ker}(\beta)} \cong \beta(T)=P \text { whence } \operatorname{dim}_{D}\left(\frac{X}{Y}\right)=1 .
$$


Hence, if we choose $x \in X-Y$, then $X=x D \oplus Y$ as $D$-spaces so that

$$
T=x D \oplus Y \oplus P=x D \oplus \operatorname{ker}(\beta) .
$$

Write $V_{D}=x D \oplus M$, for some subspace $M \supseteq \operatorname{ker}(\beta)$. Then Condition $\mathrm{S}$ shows that $v_{\circ} \in V$ exists such that $v_{\circ} M=0$ and $v_{\circ} x \neq 0$. Thus $P=D v_{\circ} x$ because $\operatorname{dim}\left({ }_{D} P\right)=1$. Write $\beta(x)=d_{\circ} v_{\circ} x$, where $d_{\circ} \in D$. Hence

$$
\beta(x d+y+p)=\beta(x d)=\beta(x) d=\left(d_{\circ} v_{\circ} x\right) d=d_{\circ} v_{\circ}(x d+y+p)
$$

because $v_{\circ} y \in v_{\circ} Y \subseteq v_{\circ} M=0$. Thus $\beta=\left(d_{\circ} v_{\circ}\right)$, which completes the proof of (1).

Question 1. If $D$ is a division ring, and $R=[D, V, P]$ is right mininjective and satisfies Condition $S$, does it follow that $R$ is right selfinjective?

In view of Proposition 2, this asks: if Condition $\mathrm{S}$ holds, $l_{V}(V)=0$, and $\operatorname{dim}\left({ }_{D} P\right)=1$, does it follow that $\operatorname{dim}\left(P_{D}\right)=1$ ? Note that if this is true then $R$ is also left mininjective because Condition $\mathrm{S}$ implies that $r_{V}(V)=0$. Note further that both $l_{V}(V)=0$ and $\operatorname{dim}\left({ }_{D} P\right)=1$ hold if and only if $R_{R}$ is uniform (Proposition 8 below).

Theorem 1 provides a vector space condition that the Faith conjecture is false.

Theorem 2. Suppose that there exists a bimap $V \times V \rightarrow P$ over a division ring $D$ such that.

(1) $l_{V}(V)=0$ and $\operatorname{dim}\left({ }_{D} P\right)=1=\operatorname{dim}\left(P_{D}\right)$.

(2) Condition $S$ holds.

(3) $\operatorname{dim}\left(V_{D}\right)=\infty$.

Then the Faith conjecture is false.

Proof. $R=[D, V, P]$ is local with $J^{3}=0$, by Lemma 1 , and $R$ is right selfinjective by Theorem 1 . However, $R$ is not right artinian by Proposition 1 .

Note that if (1) and (2) in Theorem 2 hold, the proof shows that $R[D, V, P]$ is a counterexample to the Faith conjecture if and only if $\operatorname{dim}\left(V_{D}\right)=\infty$. In Theorem 3 below we give some matrix conditions that $R[D, V, P]$ is a counterexample to the conjecture.

\section{Question 2. Is there a converse to Theorem 2?}

Some examples. Thus the Faith conjecture is related to the existence of certain bimaps, and the following two results reveal one aspect of the structure of these bimaps. Recall that $\operatorname{hom}\left(V_{D}, P_{D}\right)$ is a $D$ - $D$-bimodule via

$$
\left.\begin{array}{l}
(d \lambda)(v)=d \lambda(v) \\
(\lambda d)(v)=\lambda(d v)
\end{array}\right\} \text { for all } \lambda \in \operatorname{hom}\left(V_{D}, P_{D}\right), d \in D \text { and } v \in V .
$$

The next proposition isolates the conditions $\mathrm{S}$ and $l_{V}(V)=0$ occurring in Theorem 1. 
Proposition 3. Let $D$ be a division ring, let ${ }_{D} V_{D}$ and ${ }_{D} P_{D}$ be bimodules, and assume that $\operatorname{dim}\left({ }_{D} P\right)=1=\operatorname{dim}\left(P_{D}\right)$. Given a bimap $V \times V \rightarrow P$ define

$$
\sigma:{ }_{D} V_{D} \rightarrow \operatorname{hom}\left(V_{D}, P_{D}\right) \text { by } \sigma(v)=v \cdot \text { for all } v \in V \text {. }
$$

Then $\sigma$ is a D-D-bimodule homomorphism and

(1) $\sigma$ is one-to-one if and only if $l_{V}(V)=0$,

(2) $\sigma$ is onto if and only if Condition $\mathrm{S}$ holds.

Proof. It is routine to check that $\sigma$ is a bimodule homomorphism and so (1) follows from the fact that $\operatorname{ker}(\sigma)=\{u \mid u V=0\}=l_{V}(V)$.

To prove (2), assume first that Condition $\mathrm{S}$ holds and let $\lambda \in \operatorname{hom}\left(V_{D}, P_{D}\right)$. If $\lambda=0$ then $\lambda=\sigma(0)$. If $\lambda \neq 0$ use the fact that $\operatorname{dim}\left(P_{D}\right)=1$ to write $V=x D \oplus \operatorname{ker}(\lambda)$. By Condition S let $v_{\circ} \in V$ satisfy $v_{\circ} x \neq 0$ and $v_{\circ} \operatorname{ker}(\lambda)=0$. Fix $0 \neq p_{\circ} \in P$ so that $P=D p_{\circ}$. Write $v_{\circ} x=d_{\circ} p_{\circ}$ and $\lambda(x)=d_{1} p_{\circ}$, where $d_{\circ}$ and $d_{1}$ are in $D$. If $v_{1}=d_{1} d_{\circ}^{-1} v_{\circ}$, then $v_{1} x=d_{1} p_{\circ}=\lambda(x)$ while, for $k \in \operatorname{ker}(\lambda)$, $v_{1} k=d_{1} d_{\circ}^{-1} v_{\circ} k=0=\lambda(k)$. Since $V=x D \oplus \operatorname{ker}(\lambda)$, this shows that $\lambda=v_{1} \cdot=\sigma\left(v_{1}\right)$. Conversely, if $V=x D \oplus M$ and $P=D p_{\circ}$, define $\lambda: V_{D} \rightarrow P_{D}$ by $\lambda(x d+m)=p_{\circ} d$. If $\sigma$ is onto, let $\lambda=v_{\circ}$. where $v_{\circ} \in V$. Then $v_{\circ} x=\lambda(x)=p_{\circ} \neq 0$ and $v_{\circ} M=\lambda(M)=0$. This proves Condition $\mathrm{S}$.

Thus, if $R=[D, V, P]$ is right selfinjective and $\left\{v_{i} \mid i \in I\right\}$ is a basis of $V_{D}$, then

$$
{ }_{D} V_{D} \cong \operatorname{hom}\left(V_{D}, P_{D}\right)=\operatorname{hom}\left(\oplus_{i \in I} v_{i} D, P_{D}\right) \cong \Pi_{i \in I} \operatorname{hom}\left(v_{i} D, P\right)
$$

so that, as $\operatorname{dim}\left(P_{D}\right)=1$, we have $|V| \geq 2^{|I|}$.

The set of all bimaps $\varphi: V \times V \rightarrow P$ becomes a $\mathbb{Z}$-bimodule using pointwise operations, where $\mathbb{Z}$ denotes the integers. Proposition 3 reveals that there is a close connection between the bimaps $V \times V \rightarrow P$ and $h o m\left(V_{D}, P_{D}\right)$. In fact there is a $\mathbb{Z}$ isomorphism.

Proposition 4. If $\varphi: V \times V \rightarrow P$ is a bimap, define $\varphi^{\prime}: V \rightarrow \operatorname{hom}\left(V_{D}, P_{D}\right)$ by $\varphi^{\prime}(v)=v \cdot$. Then $\varphi^{\prime}$ is $D$-D-linear, and $\varphi \rightarrow \varphi^{\prime}$ is a $\mathbb{Z}$-isomorphism

$$
\{\text { bimaps } \varphi: V \times V \rightarrow P\} \rightarrow\left\{D \text {-D-morphisms } \theta:{ }_{D} V_{D} \rightarrow \operatorname{hom}\left(V_{D}, P_{D}\right)\right\}
$$

with inverse $\theta \mapsto \theta^{\prime}$, where $\theta^{\prime}(v, w)=[\theta(v)](w)$ for all $v$ and $w$ in $V$.

Proof. We omit the routine verifications.

Now let $V=D^{(I)}$ be the direct sum of $|I|$ copies of $D$, and write $v \in V$ as $v=\left\langle v_{i}\right\rangle$, thought of as a row vector. If $A=\left[a_{i j}\right]$ is any $I \times I$ matrix over $D$, then

$$
v A=<\Sigma_{i} v_{i} a_{i j}>\text { and } A v^{T}=<\Sigma_{j} a_{i j} v_{j}>
$$

are both defined (but lie in the direct product $D^{I}$ ). Hence we may define a product $V \times V \rightarrow D$ by

$$
v w=v A w^{T}=\Sigma_{i, j} v_{i} a_{i j} w_{j}
$$


This satisfies the axioms for a bimap except possibly for $(v d) w=v(d w)$, and this latter requirement holds if and only if each $a_{i j}$ lies in the center of the division ring $D$. In fact the condition $(v d) w=v(d w)$ means $\Sigma_{i, j} v_{i}\left(d a_{i j}\right) w_{j}=\Sigma_{i, j} v_{i}\left(a_{i j} d\right) w_{j}$, for all $v_{i}$ and $w_{j}$, which implies that $d a_{i j}=a_{i j} d$. Furthermore, every bimap into $D$ arises in this way. Indeed, if $\left\{e_{i} \mid i \in I\right\}$ is the standard basis of $D^{(I)}$ then $a_{i j}=e_{i} e_{j}$ is central in $D$ and $v w=\left(\Sigma_{i} v_{i} e_{i}\right)\left(\Sigma_{j} e_{j} w_{j}\right)=v A w^{T}$.

Example 1 . Let $I=\{1,2, \cdots\}$ and, given $n \geq 1$, let $A$ be the $I \times I$ matrix where the first $n$ rows are zero and the remaining rows are a copy of the $I \times I$ identity matrix. Thus $v w=v_{n+1} w_{1}+v_{n+2} w_{2}+\cdots$, so that $r_{V}(V)=0$ while we have

$$
l_{V}(V)=\left\{<u_{1}, u_{2}, \cdots, u_{n}, 0,0, \cdots>\mid u_{i} \in V\right\} \text { has dimension } n .
$$

Example 2. Again let $I=\{1,2, \cdots\}$ but now let $A$ be the $I \times I$ matrix where the even rows are zero and the odd rows are the rows of the $I \times I$ identity matrix in order. Thus $v w=v_{1} w_{1}+v_{3} w_{2}+v_{5} w_{3}+\cdots$. In this case we have $r_{V}(V)=0$ but $l_{V}(V)=\left\{<0, u_{2}, 0, u_{4}, 0, u_{6}, \cdots>\mid u_{i} \in V\right\}$ has infinite dimension.

Example 3. Let $V=D^{n}$ and let $A$ be an $n \times n$ matrix from the center of $D$. Then $v w=v A w^{T}$ is a bimap $V \times V \rightarrow D$ as above, and the following are equivalent for $R=[D, V, D]$ :

(1) $R$ is quasi-Frobenius,

(2) $R$ is right selfinjective,

(3) $R$ is right mininjective,

(4) $A$ is invertible.

Indeed, it is clear that $(1) \Rightarrow(2) \Rightarrow(3)$. It is a routine matter to verify that $l_{V}(V)=0$ if and only if $v A=0$ implies $v=0$; that is if and only if $A$ is invertible. Thus $(3) \Rightarrow(4)$ by Proposition 2 because $P=D$ here. Finally, $R$ is artinian by Proposition 1 and so, if $A$ is invertible, (1) follows if we can prove (2). By Theorem 1, we need only verify Condition $\mathrm{S}$. Let $V=x_{1} D \oplus M_{D}$ and $\left\{x_{2}, \cdots, x_{n}\right\}$ be a basis of $M_{D}$. Then $B=\left[x_{1}^{T}, \cdots, x_{n}^{T}\right]$ is an invertible matrix. Let $v_{\circ}=[1,0, \cdots, 0] B^{-1} A^{-1}$. Then

$$
[1,0, \cdots, 0]=v_{\circ} A B=v_{\circ}\left[A x_{1}^{T}, \cdots, A x_{n}^{T}\right]=\left[v_{\circ} x_{1}, \cdots, v_{\circ} x_{n}\right]
$$

so that $v_{\circ} x_{1} \neq 0$ and $v_{\circ} M=0$. Thus $(4) \Rightarrow(1)$.

More generally, we can identify matrix conditions needed to construct a counterexample to the Faith conjecture. Let ${ }_{D} V$ be any $D$-space with basis $\left\{e_{i} \mid i \in I\right\}$ where $I$ is infinite, and let $\operatorname{RFM}_{I}(D)$ denote the ring of all row-finite $I \times I$ matrices over $D$. Given a bimodule structure ${ }_{D} V_{D}$ on $V$ we obtain a ring homomorphism $\rho: D \rightarrow \operatorname{RFM}_{I}(D)$ given for $d \in D$ by

$$
\rho(d)=\left[\rho_{i j}(d)\right], \text { where } e_{i} d=\Sigma_{k \in I} \rho_{i k}(d) e_{k} .
$$

Conversely, every bimodule structure ${ }_{D} V_{D}$ arises in this way from such a representation $\rho$.

Given $\rho$ we get a bimodule ${ }_{D} V_{D}$ so, if $\left\{f_{k} \mid k \in K\right\}$ is a basis of $V_{D}$, we obtain the "adjoint" representation $\psi: D \rightarrow C F M_{K}(D)$, the column finite matrices, given for $d \in D$ by 


$$
\psi(d)=\left[\psi_{i j}(d)\right], \text { where } d f_{k}=\Sigma_{l \in K} f_{l} \psi_{l k}(d) .
$$

If $A \in M_{I \times K}(D)$ is an arbitrary $I \times K$ matrix, we get a product $V \times V \rightarrow D$, written $(v, w) \mapsto v \cdot w$, given by

$$
v \cdot w=\Sigma_{i, k} v_{i} a_{i k} w_{k}, \text { where } v=\Sigma_{i} v_{i} e_{i} \text { and } w=\Sigma_{k} f_{k} w_{k} .
$$

As before, this satisfies all the bimap axioms except possibly $(v d) w=v(d w)$. Since $e_{i} \cdot f_{k}=a_{i k}$ we have

$$
\left(e_{i} d\right) \cdot f_{k}=e_{i} \cdot\left(d f_{k}\right) \text { if and only if } \Sigma_{j} \rho_{i j}(d) a_{j k}=\Sigma_{m} a_{i m} \psi_{m k}(d) .
$$

It follows that (1) defines a bimap on ${ }_{D} V_{D}$ if and only if

$$
\rho(d) A=A \psi(d), \text { for all } d \in D .
$$

Theorem 3. Given a bimodule ${ }_{D} V_{D}$, let $\left\{e_{i} \mid i \in I\right\}$ and $\left\{f_{k} \mid k \in K\right\}$ be bases of ${ }_{D} V$ and $V_{D}$ respectively, and assume that an $I \times K$ matrix $A$ satisfies $\rho(d) A=A \psi(d)$, for all $d \in D$, as above. Then the following are equivalent.

(i) $R=[D, V, D]$ is a counterexample to the Faith conjecture.

(ii) The rows of $A$ are a basis of the direct product $D^{K}$.

Proof. In view of Theorem 2, it suffices to prove the following statements.

(a) $l_{V}(V)=0$ if and only if the rows of $A$ are independent.

(b) Condition $\mathrm{S}$ is satisfied if and only if the rows of $A$ span ${ }_{D}\left(D^{K}\right)$.

Given $v=\Sigma_{i} v_{i} e_{i}$ in $V$ write $\bar{v}=<v_{i}>\in D^{(I)}$. Observe that $v \cdot f_{k}=\Sigma_{i} v_{i}\left(e_{i} \cdot f_{k}\right)=$ $\Sigma_{i} v_{i} a_{i j}$, so that

$$
<v \cdot f_{k}>=\bar{v} A \text {. }
$$

Hence if $v \in V$, then $v \cdot V=0$ if and only if $v \cdot f_{k}=0$, for all $k \in K$, if and only if $\bar{v} A=0$. Now (a) follows because the rows of $A$ are independent if and only if $\bar{v} A=0$ implies $\bar{v}=0$.

If Condition $\mathrm{S}$ holds and $0 \neq \bar{b}=<b_{k}>\in D^{K}$ is given, let $P \in C F M_{K}(D)$ be an invertible matrix with $\bar{b}$ as row 0 . Define

$$
<f_{k}^{\prime}>=<f_{k}>P^{-1},
$$

so that $\left\{f_{k}^{\prime} \mid k \in K\right\}$ is a basis of $V_{D}$. By Condition S let $v_{0} \in V$ satisfy

$$
v_{0} \cdot f_{k}^{\prime}= \begin{cases}1 & \text { if } k=0 \\ 0 & \text { if } k \neq 0 .\end{cases}
$$

Then observe that

$$
v_{0} \cdot f_{k}=v_{0} \cdot\left(\Sigma_{l} f_{l}^{\prime} p_{l k}\right)=\Sigma_{l}\left(v_{0} \cdot f_{l}^{\prime}\right) p_{l k}=p_{0 k}=b_{k} .
$$

Hence (3) shows that $\bar{b}=\bar{v}_{0} A$ is a linear combination of the rows of $A$.

Finally, assume that the rows of $A$ span ${ }_{D}\left(D^{K}\right)$. If $\left\{f_{k}^{\prime} \mid k \in K\right\}$ is any basis of $V_{D}$ it suffices to find $v_{0} \in V$ such that (4) holds. If $\bar{e}_{0}$ is row 0 of the $K \times K$ identity 
matrix, this asks for $v_{0} \in V$ such that $\bar{e}_{0}=<v_{0} \cdot f_{k}^{\prime}>$. But there exists an invertible matrix $P \in C F M_{K}(D)$ such that $<f_{k}>=<f_{k}^{\prime}>P$. By hypothesis row 0 of $P$ is a linear combination of the rows of $A$; that is $\bar{e}_{0} P=\bar{v}_{0} A$, for some $v_{0} \in V$. But then (3) gives

$$
\bar{e}_{0} P=\bar{v}_{0} A=<v_{0} \cdot f_{k}>=<v_{0} \cdot f_{k}^{\prime}>P
$$

using the fact that $\left\langle f_{k}\right\rangle=<f_{k}^{\prime}>P$. Since $P$ is invertible, $\bar{e}_{0}=<v_{0} \cdot f_{k}>$ as required.

One difficulty with applying Theorem 3 is that, for a bimodule ${ }_{D} V_{D}$, we cannot define the map $\rho$ in terms of $A$ and $\psi$. In a concrete example we have to first find $\rho$ and $\psi$ and then ask for the matrix $A$. However $A$ need not exist in general, even in the finite dimensional case. For example, let $D=F$ be a commutative field with endomorphism $\sigma: F \rightarrow F$, and consider $V=F^{n}$, where the right structure $V_{F}$ is as usual, and the left structure is defined by $f \cdot v=\sigma(f) v$. Then an invertible $A$ exists such that (2) is satisfied if and only if $\sigma^{2}=1_{F}$. This example illustrates that the structure of $A$ depends heavily on the particular bimodule structure, and not only on the dimensions.

Other Properties of $R=[D, V, P]$. Many other properties of the ring $R=[D, V, P]$ can be characterized as in Theorem 1 in terms of vector space properties of $V$ and $P$. Several of these are collected in this section.

A ring $R$ is called right Kasch if every simple right $R$-module embeds in $R_{R}$. The ring $R=[D, V, P]$ is local and so has only one simple module. Since $P \neq 0$ we have $\operatorname{soc}\left(R_{R}\right) \neq 0$ (and $\operatorname{soc}\left({ }_{R} R\right) \neq 0$ ) by Lemma $1(4)$, whence we have the following result.

Proposition 5. $R=[D, V, P]$ is right and left Kasch.

The next result follows from Lemma 1(4) and the fact that $\operatorname{soc}\left(R_{R}\right) \subseteq{ }^{e s s} R_{R}$.

Proposition 6. $R=[D, V, P]$ has finite right uniform dimension if and only if $\operatorname{dim}\left(P_{D}\right)<\infty$ and $\operatorname{dim}\left[l_{V}(V)_{D}\right]<\infty$.

A ring $R$ is called a left minannihilator ring if $\operatorname{lr}(K)=K$, for all simple left ideals $K$. These rings are closely related to the right mininjective rings (see [8]) and the following result shows that if $R=[D, V, P]$ is left minannihilator then it is right mininjective.

Proposition 7. The following are equivalent for $R=[D, V, P]$.

(1) $R$ is a left minannihilator ring.

(2) $l_{V}(V)=0=r_{V}(V)$ and $\operatorname{dim}\left({ }_{D} P\right)=1$.

(3) $\operatorname{soc}\left(R_{R}\right)=\operatorname{soc}\left({ }_{R} R\right)$ is simple as a left $R$-module.

Proof. (1) $\Rightarrow(2)$. If $0 \neq p \in P$, then $r(p) \supseteq r(P)=J$ and so $r(p)=J$ because $R$ is local. As $D p=R p$ is simple, (1) gives

$$
D p=\operatorname{lr}(p)=l(J)=\operatorname{soc}\left(R_{R}\right)=l_{V}(V) \oplus P .
$$


As $P \neq 0$, this gives $l_{V}(V)=0$ and $\operatorname{dim}\left({ }_{D} P\right)=1$. Finally, if $w \in r_{V}(V)$ and $0 \neq p \in P$, then $w+p$ and $p$ are in $\operatorname{soc}\left({ }_{R} R\right)$ so that $r(w+p)=J=r(p)$. As before, (1) gives $D(w+p)=\operatorname{lr}(w+p)=\operatorname{lr}(p)=D p$. Since $V \oplus P$ is direct, this implies that $w=0$, whence $r_{V}(V)=0$.

$(2) \Rightarrow(3)$. Using Lemma 1(4), $\operatorname{soc}\left({ }_{R} R\right)=r_{V}(V) \oplus P=P=l_{V}(V) \oplus P=\operatorname{soc}\left(R_{R}\right)$. This is left simple because $\operatorname{dim}\left({ }_{D} P\right)=1$.

(3) $\Rightarrow(1)$. Write $S=\operatorname{soc}\left({ }_{R} R\right)=\operatorname{soc}\left(R_{R}\right)$. This the only simple left ideal by (3), so that $S=P$ and (1) follows from $\operatorname{lr}(S)=l(J)=\operatorname{soc}\left(R_{R}\right)=S$.

A ring $R$ is said to satisfy the right $\mathrm{C} 1$-condition if every right ideal of $R$ is essential in a summand $e R, e^{2}=e$. The right $\mathrm{C} 2$-condition holds in $R$ if every right ideal of $R$ that is isomorphic to a summand is itself a summand. A ring is called right continuous if it satisfies both the right $\mathrm{C} 1$-condition and the right $\mathrm{C} 2$-condition. Clearly every right selfinjective ring is right continuous.

Proposition 8. Let $R=[D, V, P]$.

(1) $R$ always satisfies the left and right $\mathrm{C} 2$-conditions.

(2) The following are equivalent.

(a) $R$ is right continuous.

(b) $R_{R}$ is uniform.

(c) $\operatorname{soc}\left(R_{R}\right)$ is simple.

(d) $l_{V}(V)=0$ and $\operatorname{dim}_{D}\left(P_{D}\right)=1$.

(e) $P \subseteq T$ for all right ideals $T \neq 0$.

(f) Every right ideal $T \neq 0, R$ has the form $T=X \oplus P$, where $X_{D} \subseteq V_{D}$.

Proof. Let $T \cong e R, e^{2}=e$. As $R$ is local, either $e=0$ (so that $T=0$ is a summand) or $e=1$. In the last case, $T=a R$, where $a \in R$ and $r(a)=0$. Thus $a \notin J$ and so $T=R$ is a summand. This proves half of (1); the rest follows by symmetry. is local.

(a) $\Rightarrow$ (b). If $T \neq 0$ is a right ideal then $T \subseteq{ }^{\text {ess }} R_{R}$ by the C1-condition because $R$

(b) $\Rightarrow$ (c). This is clear since $\operatorname{soc}\left(R_{R}\right) \neq 0$ by our standing assumption that $P \neq 0$.

(c) $\Rightarrow(d)$. This follows from (4) and (7) of Lemma 1 because $P \neq 0$.

(d) $\Rightarrow\left(\right.$ e). Suppose that $T \neq 0$ and $P \nsubseteq T$. Then $T \cap P=0$ because $\operatorname{dim}_{D}\left(P_{D}\right)=1$. We may assume that $T \subseteq J$ because $R$ is local. Let $t=v+p \in T$. If $v_{1} \in V$ we have $t v_{1}=v v_{1} \in T \cap P=0$, and so $v \in l_{V}(V)=0$. Thus $T \subseteq P$, a contradiction.

(e) $\Rightarrow(f)$. This is clear from Lemma $1(6)$.

(f) $\Rightarrow$ (a). If $T \neq 0$ is a right ideal, then $0 \neq P \subseteq T$, by (f). It follows that $R_{R}$ is uniform, so that $T \subseteq \subseteq^{\text {ess }} R_{R}$. Hence $R$ satisfies the C1-condition and so (a) follows from (1).

We now turn to a discussion of annihilators. Observe first that the following statements are valid.

If $X_{D}=r_{V}(Y)$, where $Y \subseteq V$, we may assume that $Y={ }_{D} Y$ because $X=r_{V}\left[l_{V} r_{V}(Y)\right]$. If ${ }_{D} X=l_{V}(Y)$, where $Y \subseteq V$, we may assume that $Y=Y_{D}$ because $X=l_{V}\left[r_{V} l_{V}(Y)\right]$.

Lemma 4. Let $R=[D, V, P]$.

(1) If $T=X_{D} \oplus P$, where $X \subseteq V$, then $l(T)=l_{V}(X) \oplus P$.

(2) If $L={ }_{D} Y \oplus P$, where $Y \subseteq V$, then $r(L)=r_{V}(Y) \oplus P$. 
Proof. We prove (1); (2) is similar. We have $l(T) \subseteq J$ as $T \neq 0$. If $v+p \in l(T)$, then $v x=(v+p) x=0$, for all $x \in X$; that is $v \in l_{V}(X)$. Thus $l(T) \subseteq l_{V}(X) \oplus P$. Conversely, if $v \in l_{V}(X)$ then $(v+p)\left(x+p_{1}\right)=v x=0$, for all $x+p_{1}$ in $T$, and so $l_{V}(X) \oplus P \subseteq l(T)$.

Lemma 5. Let $R=[D, V, P]$ and suppose $T \neq 0$ and $L \neq 0$ are proper right and left ideals of $R$ respectively.

(1) $T$ is a right annihilator in $R$ if and only if $T=r_{V}(Y) \oplus P$, for some ${ }_{D} Y \subseteq V$.

(2) $L$ is a left annihilator in $R$ if and only if $L=l_{V}(X) \oplus P$, for some $X_{D} \subseteq V$.

Proof. Again we prove only (1), as (2) is analogous. If $T=r_{V}(Y) \oplus P$, then $T=r(Y \oplus P)$, by Lemma 4. Conversely, if $T$ is a right annihilator, then $T=r l(T)$. Now $T \neq R$ means $T \subseteq J$ and so $P \subseteq l(T)$. Hence $l(T)=Y \oplus P$, for some ${ }_{D} Y \subseteq V$, by Lemma 1(6), so that $T=r l(T)=r(Y \oplus P)=r_{V}(Y) \oplus P$, by Lemma 4 .

We say that $V$ has $A C C$ on left annihilators if it has ACC on subspaces of the form $l_{V}(X)$, where $X \subseteq V$, with similar terminology for the DCC and for right annihilators.

Proposition 9. Let $R=[D, V, P]$. Then $R$ has ACC (DCC) on right (left) annihilators if and only if the same is true for $V$.

Proof. We give the argument for the ACC on right annihilators; the other three cases are analogous. By Lemma 5, every ascending chain of right annihilators in $R$ has the form $r_{V}\left(Y_{1}\right) \oplus P \subseteq r_{V}\left(Y_{2}\right) \oplus P \subseteq \cdots$. This gives $r_{V}\left(Y_{1}\right) \subseteq r_{V}\left(Y_{2}\right) \subseteq \cdots$ and so, if $V$ has the ACC, $r_{V}\left(Y_{n}\right)=r_{V}\left(Y_{n+1}\right)=\cdots$ for some $n$. Hence the chain in $R$ terminates. Conversely, if $r_{V}\left(Y_{1}\right) \subseteq r_{V}\left(Y_{2}\right) \subseteq \cdots$ in $V$, then $r\left(Y_{1} \oplus P\right) \subseteq$ $r\left(Y_{2} \oplus P\right) \subseteq \cdots$ by Lemma 4. If $r\left(Y_{n} \oplus P\right)=r\left(Y_{n+1} \oplus P\right)=\cdots$ for some $n$, it follows from Lemma 4 that $r_{V}\left(Y_{n}\right)=r_{V}\left(Y_{n+1}\right)=\cdots$.

Using Lemma 2, we can locate the right singular ideal $Z\left(R_{R}\right)$ in $R=[D, V, P]$.

Proposition 10. Let $R=[D, V, P]$ and assume that $\operatorname{dim}\left(P_{D}\right)=1$.

(1) $\left.Z\left(R_{R}\right)=l_{V} l_{V}(V) \oplus P=\| \operatorname{soc}\left(R_{R}\right)\right] \subseteq \subseteq^{e s s} R_{R}$.

(2) $\operatorname{soc}\left(R_{R}\right) \subseteq Z\left(R_{R}\right)$.

(3) $Z\left(R_{R}\right)=J$ if and only if $l_{V}(V) \subseteq r_{V}(V)$ if and only if $\operatorname{soc}\left(R_{R}\right) \subseteq \operatorname{soc}\left({ }_{R} R\right)$.

Proof. For convenience write $U=l_{V}(V)$, so that $\operatorname{soc}\left(R_{R}\right)=U \oplus P$, by Lemma 1(4).

(1). Always $Z\left(R_{R}\right) \subseteq l\left[\operatorname{soc}\left(R_{R}\right)\right]=l_{V}(U) \oplus P$. We claim that $l_{V}(U) \oplus P \subseteq Z\left(R_{R}\right)$. Let $y=v+p \in l_{V}(U) \oplus P$. Since $v \in l_{V}(U)$ we have $U \subseteq r_{V}(v)$, and so $\operatorname{soc}\left(R_{R}\right)=U \oplus P \subseteq r_{V}(v) \oplus P=r(y)$. Thus $y \in Z\left(R_{R}\right)$ because $\operatorname{soc}\left(R_{R}\right) \subseteq{ }^{\text {ess }} R_{R}$. This proves the equalities in (1). Finally, $U \subseteq l_{V}(U)$ because $U^{2}=0$. Hence $\operatorname{soc}\left(R_{R}\right) \subseteq l_{V}(U) \oplus P=l\left[\operatorname{soc}\left(R_{R}\right)\right]$, and (1) follows.

(2). Since $U^{2}=0$ we have $\left[\operatorname{soc}\left(R_{R}\right)\right]^{2}=0$, so that $\operatorname{soc}\left(R_{R}\right) \subseteq l\left[\operatorname{soc}\left(R_{R}\right)\right]$ and (2) follows from (1).

(3). Since $Z\left(R_{R}\right)=l_{V}(U) \oplus P$ and $J=V \oplus P$, we have $Z\left(R_{R}\right)=J$ if and only if $l_{V}(U)=V$ if and only if $V U=0$ if and only if $U \subseteq r_{V}(V)$. The second equivalence holds because $\operatorname{soc}\left(R_{R}\right)=U \oplus P$ and $\operatorname{soc}\left({ }_{R} R\right)=r_{V}(\bar{V}) \oplus P$ (by the right-left analogue of Lemma 1(4)). 
A ring $R$ is called right principally injective (right $P$-injective) [7] if every $R$-linear map from a principal right ideal of $R$ to $R$ is given by left multiplication by an element of $R$, equivalently if $\operatorname{lr}(a)=R a$ for all $a \in R$. These rings are both right mininjective and left minannihilator, a fact which is reflected in the following result.

Proposition 11. If $R=[D, V, P]$, then $R$ is right P-injective if and only if it satisfies the following three conditions:

(a) $\operatorname{dim}\left({ }_{D} P\right)=1$,

(b) $l_{V}(V)=0=r_{V}(V)$,

(c) $l_{V} r_{V}(v)=D v$ for all $v \in V$.

Proof. Assume first that $R$ is right P-injective. Then Proposition 2 implies (a) and $l_{V}(V)=0$. To show that $r_{V}(V)=0$, suppose that $0 \neq w \in r_{V}(V)$. Then $V w=0$ so that $R w=D w$, and we have $\operatorname{lr}(w)=R w=D w \subseteq V$, by P-injectivity. But if $p \in P$, then $r(w) \subseteq J=r(p)$ and so $p \in \operatorname{lr}(w)$. This implies that $P \subseteq V$, a contradiction. Hence $r_{V}(V)=0$, proving (b).

Claim. If $0 \neq v \in V$ and $p \in P$, then $R(v+p)=D v \oplus P$.

Proof. Observe first that $V v=P$ by (a) because $v \notin r_{V}(V)$. Hence

$$
R(v+p)=\left\{d v+\left(d p+v_{1} v\right) \mid d \in D \text { and } v_{1} \in V\right\}=D v \oplus P,
$$

proving the Claim.

To show that $D v=l_{V} r_{V}(v)$, we may assume that $v \neq 0$. Then the Claim and Lemma 4 give

$$
r(v)=r(R v)=r[D v \oplus P]=r_{V}(v) \oplus P .
$$

Hence

$$
l_{V} r_{V}(v) \oplus P=\operatorname{lr}(v)=R v=D v \oplus P
$$

and (c) follows.

Conversely, assume (a), (b) and (c). If $a \in R$ we must show that $\operatorname{lr}(a)=R a$. This is clear if $a=0$ or if $a \notin J$ (because $R$ is local), and it also holds if $a \in P$; (then $r(a)=J=V \oplus P$, so that $\operatorname{lr}(a)=l_{V}(V) \oplus P=P=R a$ by (b)). Assume $a \in J-P$, say $a=v+p$, where $v \neq 0$. Then $R a=D v \oplus P$ by the Claim (the proof uses only $\operatorname{dim}\left({ }_{D} P\right)=1$ and $r_{V}(V)=0$ ) and so Lemma 4 (twice) gives $r(a)=r_{V}(v) \oplus P$. Hence $\operatorname{lr}(a)=l_{V} r_{V}(v) \oplus P=D v \oplus P=R a$ by (c).

Example 4. As in Examples 1, 2 and 3 above, let $D=D^{(I)}$, where $I=\{1,2,3, \cdots\}$. If $A$ is the $I \times I$ identity matrix, the bimap is $v w=v_{1} w_{1}+$ $v_{2} w_{2}+\cdots$, where $v=\left\langle v_{i}>\right.$ and $w=\left\langle w_{i}\right\rangle$. Then $l_{V}(V)=0=r_{V}(V)$ is clear and it is a routine matter to verify that $l_{V} r_{V}(v)=D v$ and $r_{V} l_{V}(v)=v D$, for all $v \in V$. Hence $R=[D, V, D]$ is a right and left $\mathrm{P}$-injective ring that is neither right nor left artinian. 
Acknowledgement. The authors would like to thank Professor K. Varadarajan for pointing out an error in a preliminary version of this paper. This research was carried out while the second and third authors were at the Centre de Recerca Matemàtica in Barcelona, Spain, and the authors are grateful for the hospitality and financial support extended to them by the CRM. The work was also supported by NSERC Grant A8075 and by the Ohio State University. The first author was partially supported by DGYCIT grant PB95-0626 and the Comissionat per Universitats i Recerca de la Generalitat de Catalunya.

\section{REFERENCES}

1. Y. Baba and K. Oshiro, On a theorem of Fuller, J. Algebra 154 (1993), 86-94.

2. J. Clark and C. V. Huynh, A note on perfect self-injective rings, Quart. J. Math. Oxford Ser. (2) 45 (1994), 13-17.

3. J. Clark and C. V. Huynh, When is a self-injective semiperfect ring quasi-Frobenius? J. Algebra 165 (1994), 531-532.

4. C. Faith, Rings with ascending chain conditions on annihilators, Nagoya Math. J. 27 (1966), 179-191.

5. C. Faith, When self-injective rings are QF: A report on a problem, Centre Recerca Matemàtica Institut d'Estudis Catalans (Spain, 1990).

6. D. Herbera and A. Shamsuddin, On self-injective perfect rings, Canad. Math. Bull. 39 (1996), 55-58.

7. W. K. Nicholson and M. F. Yousif, Principally injective rings, J. Algebra 174 (1995), 77-93.

8. W. K. Nicholson and M. F. Yousif, Mininjective rings, J. Algebra 187 (1997), 548578.

9. W. K. Nicholson and M. F. Yousif, On perfect simple-injective rings, Proc. Amer. Math. Soc. 125 (1997), 979-985.

10. B. Osfosky, A generalization of quasi-Frobenius rings, J. Algebra 4 (1966), 373-389.

11. W. Xue, Rings related to quasi-Frobenius rings, Algebra Colloq. 5 (1998), 471-480. 